\title{
Block Milne's Implementation For Solving Fourth Order Ordinary Differential Equations
}

\author{
J. G. Oghonyon \\ Department of Mathematics \\ Covenant University \\ Ota, Nigeria \\ godwin.oghonyon@covenantuniversity.edu.ng \\ K. S. Eke \\ Department of Mathematics, \\ Covenant University \\ Ota, Nigeria \\ kanayo.eke@covenantuniversity.edu.ng
}

\author{
S. A. Okunuga \\ Department of Mathematics \\ University of Lagos \\ Akoka-Lagos, Nigeria \\ nugabola@yahoo.com \\ O. A. Odetunmibi \\ Department of Mathematics \\ Covenant University \\ Ota, Nigeria \\ wole.odetunmibi@covenantuniversity.edu.ng
}

\begin{abstract}
Block predictor-corrector method for solving non-stiff ordinary differential equations (ODEs) started with Milne's device. Milne's device is an extension of the block predictorcorrector method providing further benefits and better results. This study considers Milne's devise for solving fourth order ODEs. A combination of Newton's backward difference interpolation polynomial and numerical integration method are applied and integrated at some selected grid points to formulate the block predictor-corrector method. Moreover, Milne's devise advances the computational efficiency by applying the chief local truncation error] (CLTE) of the block predictor-corrector method after establishing the order. The numerical results were exhibited to attest the functioning of Milne's devise in solving fourth order ODEs. The complete results were obtained with the aid of Mathematica 9 kernel for Microsoft Windows. Numerical results showcase that Milne's device is more effective than existent methods in terms of design new step size, determining the convergence criteria and maximizing errors at all examined convergence levels.
\end{abstract}

Keywords-Milne's device; predictor-corrector method; suitable step size; convergence criteria; maximum errors; chief local truncation error]

\section{INTRODUCTION}

The extension of predictor-corrector method is important for providing some computational benefits to numerical integration of ordinary differential equations. These computational vantages are enlisted in $[1,2]$. This composition is primarily concerned with presenting approximate solution of fourth order ODEs of the form [1-3]:

$$
\begin{aligned}
& y^{\prime \prime \prime \prime}=f\left(x, y, y^{\prime}, y^{\prime \prime}, y^{\prime \prime \prime}\right), \\
& y(a)=\beta_{0}, y^{\prime}(a)=\beta_{1}, \\
& y^{\prime \prime}(a)=\beta_{2}, y^{\prime \prime \prime}(a)=\beta_{3}
\end{aligned}
$$

for $a \leq x \leq b$ and $f: R \times R^{\mu} \rightarrow R^{\mu}$

The numerical solution to (1) is broadly defined as

$$
\sum_{i=1}^{m} \alpha_{i} y_{n+i-1}=h^{4} \sum_{i=1}^{m} \beta_{i} f_{n+i-1}
$$

where the step size is $\mathrm{h}, \alpha_{m}=1, \alpha_{i}, \quad i=0,1, \ldots, m, \beta_{m}$ are unknown quantities which are unequivocally fixed in a way that the expression is of order $m$ as seen in $[3,4]$. It is accepted that $f \in R$ is sufficiently differentiable to a certain degree on interval $x \in[a, b]$ and meets applicable Lipchitz condition, i.e., thither is a unvarying $L \geq 0$ such that

$$
\mid f(x, y)-f(x, \bar{y}|\leq L| y-\bar{y} \mid, \quad \forall y, \bar{y} \in R .
$$

Beneath the given assumption, (1) ascertained existence and quality of singularity outlined on $a \leq x \leq b$, likewise looked at to satisfy the Weierstrass theorem $[5,6]$.

$$
\left[y_{1}^{\prime}, y_{2}^{\prime}, \ldots, y_{n}^{\prime}\right]^{T}, y=\left[y_{1}, y_{2}, \ldots, y_{n}\right] \text { and } f=\left[f_{1}, f_{2}, \ldots, f_{n}\right] \text {, }
$$

spring up in real world practical applications for state of difficulty in scientific research and applied science such as fluid dynamics and rocket motion [7].

It has been proposed that the established method of solving (1) is by reduction to first-order ODEs. This technique of simplification possesses real severe hindrance which includes waste of human exertion, complication in the use of Mathematica package/computer software for coding and squandering implementation time [2-3, 8-15]. Scholars have developed straight methods for approximating (1) with better results and efficiency. These methods include block method, block predictor-corrector method, block implicit method, block hybrid method, BDF etc.. However, there are advantages and disadvantages for implementing them. Block predictorcorrector method was hinted in [2-3, 10, 12-15]. The backward differentiation formula (BDF) is Gear's method recognized for 
stiff ODEs as seen in [7, 16-19]. The target is to employ Milne's device to develop a variable step-size block predictorcorrector method [1, 6, 20,21]. Milne's device is seen as the numerical estimation of initial value problems (IVPs) and this technic is based on numerous ingredients $[2,17]$. These include convergence criteria, predictor-corrector pattern braces of like order, designing suitable step-size and chief local truncation error. In addition, this subject field possesses a great deal of superior qualities as discoursed in [1-3, 6, 20, 21]

Definition: $b$-block, $r$ - point method. If $r$ denotes the block size and $\mathrm{h}$ is the stepsize, then block size in time is $r h$. Let $m=0,1,2, \ldots$ represent the block number and $n=m r$, then the $b$-block, $r$-point method can be written in the following general form:

$$
Y_{\mu}=\sum_{s=1}^{b} A_{s} Y_{\mu-s}+h \sum_{s=0}^{b} B_{s} F_{\mu-s}
$$

where

$$
\begin{aligned}
Y_{\mu} & =\left[y_{n+1}, \ldots, y_{n+i}, \ldots, y_{n+r}\right]^{T} \\
F_{\mu} & =\left[f_{n+1}, \ldots, f_{n+i}, \ldots, f_{n+r}\right]^{T}
\end{aligned}
$$

$A_{s}$ and $B_{s}$ are $r \times r$ coefficient matrices [16].

Therefore, from the above definition, a block method has the computational reward that in each practical application, the output is valuated to a greater extent or at more than one point concurrently. The number of points depends on the construction of the block method. Hence, utilizing this method can provide speedier solutions to the problem which can be processed to bring forth the sought-after accuracy $[2,3,7,18$, 19]. Thus, the motivation of this paper is to suggest Milne's device of variable step-size block predictor-corrector method for solving non-stiff and mildly stiff ODEs implemented in $P(E C)_{m}$ or $P(E C)_{m} E$ mode. This technique possesses the advantages like designing a suitable step size/changing the step size, specifying the convergence criteria (tolerance level) and error control/minimization likewise addressing the gaps posited above.

\section{MATERIALS AND METHODS}

To showcase this process, a variable step-size block predictor-corrector method enforcing the explicit AdamsBashforth b-step method as a predictor and the implicit AdamsMoulton b-1-step method as a corrector of the same order is prepared $[1-3,21]$. This discussion section expends the Newton's backward difference formula to prepare the block predictor-corrector method.

Presuppose $f(x)$ has a continuous $q$ th derivative, $x_{n}=x_{0}+n h$, $f_{n}=f\left(x_{n}\right)$, and backward differences are interpreted as

where $\nabla^{0} f_{n}=f_{n}$, then

$$
\nabla^{c+1} f_{n}=\nabla^{c} f_{n}-\nabla^{c} f_{n-1}
$$

$$
\begin{aligned}
& f(x)=f_{n}+\left(\frac{x-x_{n}}{h}\right) \nabla f_{n}+\left(x-x_{n}\right)\left(x-x_{n}-1\right) \frac{\nabla^{2} f_{n}}{2 ! \mathrm{h}^{2}}+ \\
& +\left(x-x_{n}\right)\left(x-x_{n}-2\right) \frac{\nabla^{3} f_{n}}{3 ! \mathrm{h}^{2}} \ldots+\left(x-x_{n}\right) \ldots(x- \\
& \left.x_{n-q+2}\right) \frac{\nabla^{q-1} f_{n}}{(q-1) ! \mathrm{h}^{q-1}}+\left(x-x_{n}\right) \ldots\left(x-x_{n-q+1}\right) \frac{\mathrm{f}^{(q)}(\varepsilon)}{q !}
\end{aligned}
$$

where $f^{(q)}(\varepsilon)$ is the qth derivative of $f$ measured at some approximate time interval owning $x, x_{n-q+1}$, and $x_{n}$. Setting $s=\frac{\left(x-x_{n-1}\right)}{h}$ and $n=m-1$, (4) turns over as

$$
\begin{aligned}
& f(x)=\left(\begin{array}{l}
-r \\
0
\end{array}\right) f_{m-1}-\left(\begin{array}{l}
-r \\
1
\end{array}\right) \nabla f_{m-1}+\ldots \\
& +(-1)^{q-1}\left(\begin{array}{l}
-r \\
q-1
\end{array}\right) \nabla^{q-1} f_{m-1}+(-1)^{q} h^{q}\left(\begin{array}{l}
-r \\
q
\end{array}\right) f^{(q)}(\varepsilon)
\end{aligned}
$$

where

$$
\left(\begin{array}{l}
r \\
s
\end{array}\right)=\frac{r(r-1) \ldots(r-s+1)}{s !} \text { and }\left(\begin{array}{l}
r \\
0
\end{array}\right)=0
$$

Interchanging the above in

$$
y\left(x_{m}\right)=y\left(t_{m-1}\right)+\int_{t_{m-1}}^{t_{m}} f(x) d x,
$$

to acquire

$$
\begin{aligned}
& y\left(x_{m}\right)=y\left(x_{m-1}\right)+\int_{x_{m}-1}^{x_{m}}\left[\sum_{i=0}^{q-1}(-1)^{i}\left(\begin{array}{c}
-r \\
i
\end{array}\right) \nabla^{i} f_{m-1}+\right. \\
& \left.(-1)^{q} h^{q}\left(\begin{array}{c}
-r \\
q
\end{array}\right) y^{(q+1)}(\varepsilon)\right] d x \\
& y(x)=y\left(x_{m-1}\right)+h \sum_{i=0}^{q-1} \varphi_{i} \nabla^{i} f_{m-1} \\
& +(-1)^{q+1} \int_{0}^{1}\left(\begin{array}{l}
-r \\
q
\end{array}\right) y^{(q+1)}(\varepsilon) d s
\end{aligned}
$$

If the terminal figure in (5) is dropped, the unexpended will be addressed with $b-$ step Adams-Bashforth formula

$$
y_{m}=y_{m-1}+h \sum_{i=0}^{q-1} \varphi_{i} \nabla^{i} f_{m-1}
$$

Establishing the backward differences in terms of the valuates at upholding points as

$$
\nabla^{d} f_{m-1}=\sum_{j=0}^{d}(-1)^{j}\left(\begin{array}{l}
d \\
j
\end{array}\right) f_{m-1-j}
$$

Equation (6) can be written as

$$
y_{m}=y_{m-1}+h \sum_{j=1}^{q} \beta_{k j} f_{m-j}
$$

where $\beta_{k j}=(-1)^{j-1} \sum_{i=j-1}^{q-1} \varphi_{i}\left(\begin{array}{c}i \\ j-1\end{array}\right)$

Equation (7) shows $y_{m}$ in terms of info at $x_{m-1}, x_{m-2}, \ldots, x_{m-q}$. Proceeding with (7) produces the block b-step Adams-Bashforth Formula [22]. Likewise, the implicit multistep method-the Adams-Moulton method can be derived by putting $n=m$ in (4) and subbing into

$$
y\left(x_{m}\right)=y\left(x_{m-1}\right)+\int_{x_{m}-1}^{x_{m}} f(x) d x
$$

to arrive at

$$
\begin{aligned}
& y\left(x_{m}\right)=y\left(x_{m-1}\right)+\int_{x_{m-1}}^{x_{m}}\left[\sum_{i=0}^{q-1}(-1)^{i}\left(\begin{array}{c}
-r+1 \\
i
\end{array}\right) \nabla^{i} f_{m}+\right. \\
& \left.(-1)^{q} h^{q}\left(\begin{array}{c}
-r+1 \\
k
\end{array}\right) y^{(q+1)}(\varepsilon)\right] d s
\end{aligned}
$$


Dropping the error term, succumbs the method as

$y_{m}=y_{m-1}+h \sum_{i=0}^{q-1} \varphi_{i}^{*} \nabla^{i} f_{m}$

Interchanging for $\nabla^{i} f_{m}$ in terms of $f_{m}, f_{m-1}, f_{m-2}, \ldots$, sets up the form

$$
y_{m}=y_{m-1}+h \sum_{j=0}^{q-1} \beta_{q j}^{*} f_{m-j}
$$

where $\beta_{q j}^{*}=(-1)^{i} h \sum_{i=j}^{q-1}\left(\begin{array}{l}i \\ j\end{array}\right) \varphi_{i}^{*}$.

In good continuation with (9), the block b-1-step AdamsMoulton Method will be brought forth [22].

\section{A. Examining Some Selected Theoretical Properties of Block Method}

Embracing [5, 6, 9, 10, 20], the consociated block methods (2) and the difference operator can be set as

$$
\begin{array}{r}
L(y(x) ; h]=\sum_{i=0}^{k} a_{i} y(x+i h) \\
-h^{4} \sum_{i=0}^{k} \beta_{\kappa} y^{\prime \prime \prime}(x+i h)
\end{array}
$$

Accepting that $y(x)$ is sufficiently and continuously differentiable on an time interval $[a, b]$ and that $y(x)$ possesses many higher derivatives as needed, then, penning the terms in (10) as a Taylor series formulation of $y\left(x_{n+i}\right)$ and $f\left(x_{n+i}\right) \equiv$ $y^{\prime \prime \prime \prime}\left(x_{n+i}\right)$ as

$$
\begin{aligned}
& y\left(x_{n+i}\right)=\sum_{i=0}^{\infty} \frac{(i h)}{i !} y^{(i)}\left(x_{n}\right) \text { and } \\
& y^{\prime \prime \prime \prime}\left(x_{n+i}\right)=\sum_{i=0}^{\infty} \frac{(i h)}{i !} y^{(i+1)}\left(x_{n}\right)
\end{aligned}
$$

Replacing (10) and (11) into (2), the next formulation is incurred as

$$
\begin{aligned}
& L[y(x) ; h]=C_{0} y(x)+C_{1} y^{\prime}(x)+\cdots+C_{p} h^{p} y^{(p)}(x)+ \\
& \cdots+C_{p+4} h^{p+4} y^{(p+4)}(x)+\cdots
\end{aligned}
$$

Holding onto $[5,6]$, the block method of (2) maintains order $\mathrm{p}$, if $C_{p+4}, p=0,1,2, \ldots, i=1,2, \ldots j$, are presented as:

$C_{p+1}, p=0,1,2, \ldots, i=1,2, \ldots j$, are given as follows:

$C_{0}=\alpha_{0}+\alpha_{1}+\alpha_{2}+\cdots+\alpha_{k}$,

$C_{1}=\alpha_{0}+2 \alpha_{1}+\alpha_{2}+\cdots+k \alpha_{k}$,

$C_{2}=\frac{1}{2 !}\left(\alpha_{0}+\alpha_{1}+\alpha_{2}+\cdots+\alpha_{k}\right)-\left(\beta_{0}+\beta_{1}+\beta_{2}+\cdots+\right.$ $\left.\beta_{k}\right)$,

$C_{q}=\frac{1}{q !}\left(\alpha_{1}+2^{q} \alpha_{2}+\cdots+k^{q} \alpha_{k}\right)-\frac{1}{(q-4) !}\left(\beta_{0}+2^{q-1} \beta_{1}+\right.$ $\left.\cdots+k^{q-1} \beta_{k}\right), q=5,4, \ldots$

So, the block method (2) is stated to sustain order $p \geq 1$ and error constants quantity established by the vector, $C_{q+4} \neq 0$. Agreeing with $[6,20]$, the block method (2) possesses order $p$ if

$L[y(x) ; h]=0 h^{p+4}, \quad C_{0}=C_{1}=C_{2}=\cdots C_{p}=C_{p+1}=$ $C_{p+2}=C_{p+3}=0, \quad C_{p+4} \neq 0$.
Consequently, $C_{p+4}$ is the error constant quantity and $C_{p+4} h^{p+4} y^{(p+4)}\left(x_{n}\right)$ is the chief local truncation error at the point $x_{n}$.

\section{B. Stability Analysis}

For properly analyzing the block method for stability, the block predictor-corrector method is renormalized and scripted as a block method displayed by the matrix finite difference equations as seen in $[5,23]$.

$$
A^{(0)} Y_{m}=A^{(1)} Y_{m-1}+h^{4}\left(B^{(0)} F_{m}+B^{(1)} F_{m-1}\right),
$$

where

$$
\begin{gathered}
Y_{m}=\left[\begin{array}{c}
y_{n+1} \\
y_{n+2} \\
\vdots \\
y_{n+r}
\end{array}\right], Y_{m-1}=\left[\begin{array}{c}
y_{n-r+1} \\
y_{n-r+2} \\
\vdots \\
y_{n}
\end{array}\right] \\
F_{m}=\left[\begin{array}{c}
f_{n+1} \\
f_{n+2} \\
\vdots \\
f_{n+r}
\end{array}\right], F_{m-1}=\left[\begin{array}{c}
f_{n-r+1} \\
f_{n-r+2} \\
\vdots \\
f_{n}
\end{array}\right] .
\end{gathered}
$$

The matrices $A^{(0)}, A^{(1)},\left(B^{(0)}, B^{(1)}\right.$ are $\mathrm{r}$ by $\mathrm{r}$ matrices while $Y_{m}, Y_{m-1}, F_{m}, F_{m-1}$ are r-vectors outlined above. In accordance with $[6,23]$, the boundary locus method is employed to ascertain the region of absolute stability of the block method in preparation to find the roots of absolute stability. Thus, subbing the test equation

$$
y^{\prime}=-\delta y \text { and } \bar{h}=h^{4} \delta^{4}
$$

into the block method (14) to arrive at

$$
\begin{aligned}
& \omega(r)=\operatorname{det}\left[r\left(A^{(0)}+B^{(0)} h^{4} \delta^{4}\right)\right. \\
& \left.-\left(A^{(1)}+B^{(1)} h^{4} \delta^{4}\right)\right]=0
\end{aligned}
$$

Replacing $\mathrm{h}=0$ in (15), the roots of the inferred equation will either be less than or equal to 1 . Therefore, definition [6] of absolutely stability is gratified. In addition, by [16], the boundary of the region of absolute stability can be gotten by stepping in (2) into

$$
\bar{h}(r)=\frac{\psi(r)}{\rho(r)}
$$

and where $r=e^{i \theta}=\cos \theta+i \sin \theta$ then after simplification together with valuating (16) within $\left[0^{\circ}, 180^{\circ}\right]$ the boundary of the region of absolute stability rests on the real axis. AdamsMoulton Method will be brought forth [22].

\section{Milne's Implementation on Block Predictor-Corrector Method}

Consorting with [1, 6, 20-21], the Milne's implementation in the $P(E C)_{m}$ or $P(E C)_{m} E$ mode turns indispensable for the explicit (predictor) and implicit (corrector) methods if both are discrete and of the same order, and this touchstones make important for the step number of the explicit method to be one step higher than that of the implicit method. Accordingly, the mode $P(E C)_{m}$ or $P(E C)_{m} E$ can be formally analyzed as succeeds for $\mu=1,2, \ldots$.

$P(E C)_{m}$ : 


$$
\begin{aligned}
& y_{n+k}^{[0]}+\sum_{i=0}^{k-1} \dot{\alpha_{i}} y_{n+i}^{[\mu]}=h^{4} \sum_{i=0}^{k-1} \beta_{i}^{\cdot} f_{n+i}^{[\mu-1]}, \\
& f_{n+k}^{[u]} \equiv f\left(x_{n+k}, y_{n+k}^{[u]}\right) \\
& y_{n+k}^{[u+1]}+\sum_{i=0}^{k=1} a_{i} y_{n+i}^{[\mu]}=h^{4} \beta_{j} f_{n+k}^{[u]}+h^{4} \sum_{i=0}^{k=1} \beta_{i} f_{n+i}^{[\mu-1]}, \\
& u=0,1, \ldots . \mu-1 \\
& P(E C)_{m} E: \\
& y_{n+k}^{[0]}+\sum_{i=0}^{k-1} \alpha_{i} y_{n+i}^{[\mu]}=h^{4} \sum_{i=0}^{k-1} \beta_{i}^{\bullet} f_{n+i}^{[\mu]}, \\
& f_{n+k}^{[u]} \equiv f\left(x_{n+k}, y_{n+k}^{[u]}\right) \\
& y_{n+k}^{[u+1]}+\sum_{i=0}^{k=1} a_{i} y_{n+i}^{[\mu]}=h^{4} \beta_{j} f_{n+k}^{[u]}+h^{4} \sum_{i=0}^{k=1} \beta_{i} f_{n+i}^{[\mu-1]}, \\
& u=0,1, \ldots . \mu-1 \\
& f_{n+j}^{[\mu]} \equiv f\left(x_{n+k}, y_{n+k}^{[\mu]}\right)
\end{aligned}
$$

Mentioning that as $m \rightarrow \infty$, the result of approximating with either modality will pitch to those applied by the mode of correcting to convergence. Moreover, predictor and corrector pair based on (2) can be implemented. $P(E C)_{m}$ or $P(E C)_{m} E$ modes are specified by (16), where $h$ is the step size. Since the predictor and corrector both have the same order $p . C_{p+4}, C_{p+4}^{*}$ are the error constant quantities of predictor and corrector respectively. The following effects are true:

\section{Proposition}

Let us assume that the predictor method gives order $p^{*}$ and the corrector method gives order $p$ engaged in $P(E C)_{m}$ or $P(E C)_{m} E$ mode, where $p_{*}^{*}, p, \mu$ are integers and $p^{*} \geq 1, p \geq 1, \mu \geq 1$. Then, when $p^{*} \geq p$ (or $p^{*}<p$ with $\mu>p-p^{*}$ ), then the predictorcorrector method gives the same order and the same PLTE as the corrector. If $p<p$ with $\mu=p-p^{*}$, then the predictor-corrector method gives the same order as the corrector, but not the same PLTE. If $p^{*}<p$ with $\mu \leq p-p$, then the predictor-corrector method gives the same order equal to $p^{*}+\mu$ (less than $p$ ). In distinction from others, when the predictor gives order $p^{*}$ and the corrector gives order $p$, PEC responds to bring forth a method of order $\mathrm{p}$. Nevertheless, the $P(E C)_{m}$ or $P(E C)_{m} E$ scheme gives always the same order and the same PLTE as discoursed in $[5,6]$.

Milne's device put forward that it is practicable to approximate the chief local truncation error of the explicit and implicit (predictor-corrector) method without approximating higher derivatives of $y(x)[1,6,20,21]$. Accepting that $p=p$, where $p^{*}$ and $p$ set the order of the explicit (predictor) and implicit (corrector) methods with the same order. Right away, for a method of order $p$, the chief local truncation errors can be scripted as

$$
C_{p+4}^{*} h^{p+4} y^{(p+4)}\left(x_{n}\right)=y\left(x_{n+k}\right)-W_{n+k}+O\left(h^{p+5}\right)
$$

Also,

$$
C_{p+4} h^{p+4} y^{(p+4)}\left(x_{n}\right)=y\left(x_{n+k}\right)-C_{n+k}+O\left(h^{p+5}\right)(19)
$$

where $W_{n+k}$ and $C_{n+k}$ are the predicted and corrected approximations which are presented by the method of order $p$ while $C_{p+4}^{*}$ and $C_{p+4}$ are autonomous of h. Dropping terms of degree $p+5$ and above, it is well-fixed to make approximates of the chief local truncation error of the method as

$$
\begin{aligned}
& C_{p+4} h^{p+4} y^{(p+4)}\left(x_{n}\right)= \\
& \frac{C_{p+4}}{C_{p+4}^{*}-C_{p+4}}\left|W_{n+k}-C_{n+k}\right|<\varepsilon
\end{aligned}
$$

Mentioning the realness that $C_{p+4} \neq C_{p+4}^{*}, W_{n+k} \neq C_{n+k}$, and $\varepsilon$ is the bound of convergence criteria. Moreover, the approximate of the chief local truncation error (20) is applied to check whether to consent the effects of the current step or to reconstruct the step with a smaller step size. The step is consented based on a test as ordained by (20) [24]. Equation (20) is the convergence criteria differently anticipated as Milne's estimate for adjusting to convergence. Even more, (20) examines the convergence criterion of the method throughout the try out valuation [24].

\section{NUMERICAL EXAMPLES}

Two problems were examined and worked out enforcing MI employing several distinguishable convergence criteria: $10^{-7}, 10^{-8}, 10^{-11}, 10^{-12}, 10^{-14}$ and $10^{-16}[8,10-14]$.

\section{A. Examined Problem 1:}

$y^{\prime \prime \prime \prime}(x)+y^{\prime \prime}(x)=0$

$y(0)=0, y^{\prime}(0)=\frac{1.1}{72-50 \pi}, y^{\prime \prime}(0)=\frac{1.1}{72-50 \pi}$,

$y^{\prime \prime \prime}(0)=\frac{-1}{144-100 \pi}$.

Solution: $y(x)=\frac{1-x-\cos x-1.2 \sin x}{144-100 \pi}$.

B. Examined Problem 2:

$y^{\prime \prime \prime \prime}(x)-4 y^{\prime \prime}(x)=0$

$y(0)=0, y^{\prime}(0)=3, y^{\prime \prime}(0)=0, y^{\prime \prime \prime}(0)=16$

$0 \leq x \leq 1$.

Solution: $y(x)=1-x+e^{2 x}-e^{-2 x}$.

\section{DISCUSSION OF RESULTS}

The final results present the functioning of the Milne's implementation for solving fourth order ODEs. The accomplished final results were obtained with the assistance of Mathematica 9 Kernel on Microsoft Windows (64-bit). Table I presents the mathematical final results of utilizing MI compared to existent methods. The steps for computing the maximum errors and ascertaining the convergence criteria (tolerance level) are set as follows:

$$
C_{p+4} h^{p+4} p+y^{(p+4)}\left(x_{n}\right) \cong \frac{C_{p+4}}{C_{p+4}^{*}}\left|P_{n+j}-C_{n+j}\right|<\varepsilon
$$


Remark that $C_{p+4}^{*} \neq C_{p+4}$ and $P_{n+j} \neq C_{n+j} . C_{p+4}^{*}$ and $C_{p+4}$ are autonomous of $\mathrm{h}$ and are the calculated chief local truncation errors of predictor and corrector method. $P_{n+j}$ and $C_{n+j}$ are the predicted and corrected estimations granted by the method of order p. Table I exhibits the mathematical resolutions for working out examined problems in the former section enforcing MI.

TABLE I. MILNE'S IMPLEMENTATION

\begin{tabular}{|c|c|c|}
\hline Method & Max error & Cover \\
\hline AS-SCMM & $1.63736 \mathrm{E}-08$ & $10^{-8}$ \\
\hline MI & $1.6798 \mathrm{E}-09$ & $10^{-8}$ \\
\hline MI & $1.21782 \mathrm{E}-09$ & \\
\hline AOSZ-SM & $2.93232452209 \mathrm{E}-12$ & $10^{-12}$ \\
\hline MI & $2.15055 \mathrm{E}-13$ & $10^{-12}$ \\
\hline AAFO-SPIBM & $3.5121472 \mathrm{E}-14$ & $10^{-14}$ \\
\hline MI & $0.47906 \mathrm{E}-15$ & $10^{-14}$ \\
\hline MI & $2.23218 \mathrm{E}-15$ & \\
\hline FSBM & $8.0491 \mathrm{E}-16$ & $10^{-16}$ \\
\hline MI & $8.13829 \mathrm{E}-18$ & $10^{-16}$ \\
\hline MI & $4.71357 \mathrm{E}-17$ & \\
\hline AEZ-SNM & $1.5897083 \mathrm{E}-07$ & $10^{-7}$ \\
\hline MI & $1.37682 \mathrm{E}-08$ & $10^{-7}$ \\
\hline MI & $1.80613 \mathrm{E}-08$ & \\
\hline AOSZ-SM & $5.14028819509 \mathrm{E}-10$ & $10^{-10}$ \\
\hline MI & $3.06153 \mathrm{E}-11$ & $10^{-10}$ \\
\hline DSOIVP & $7.218014 \mathrm{E}-11$ & $10^{-11}$ \\
\hline MI & $4.90652 \mathrm{E}-12$ & $10^{-11}$ \\
\hline
\end{tabular}

TABLE II. NOMENCLATURE

\begin{tabular}{|c|c|}
\hline MI & $\begin{array}{l}\text { Milne's Implementation for Solving 4rth Order } \\
\text { ODEs. }\end{array}$ \\
\hline Cover & Convergence criteria \\
\hline Method & Method used \\
\hline Maxerror & Magnitude of the maximum errors \\
\hline AAFO-SPIBM & $\begin{array}{c}\text { An accurate five off-step points implicit block } \\
\text { method for direct solution of 4rth order differential } \\
\text { equations [11] }\end{array}$ \\
\hline AEZ-SNM & $\begin{array}{l}\text { An efficient zero-stable numerical method for } \\
\text { 4rth-order differential equations [12] }\end{array}$ \\
\hline AOSZ-SM & $\begin{array}{l}\text { An order six zero-stable method for direct } \\
\text { solution of 4rth order ODEs [13]. }\end{array}$ \\
\hline AS-SCMM & $\begin{array}{l}\text { A six-step continuous multistep method for the } \\
\text { solution of general } 4 \text { rth order initial value problems } \\
\text { of ODEs [10]. }\end{array}$ \\
\hline DSOIVP & $\begin{array}{c}\text { Direct solution of initial value problems of 4rth } \\
\text { order ODEs [14] }\end{array}$ \\
\hline FSBM & $\begin{array}{c}\text { Five step block method for the solution of 4rth } \\
\text { order ODEs [8] }\end{array}$ \\
\hline
\end{tabular}

\section{A. Algorithm}

A composed algorithmic program that will blueprint a new step size and assess the maximum errors of the predictorcorrector method in the word form of $P(E C)_{m}$ or $P(E C)_{m} E$ mode, if the mode is run $m$ times.

Step 1: Choose a step size for $h$.

Step 2: The order of the predictor-corrector method must be the same.

Step 3: The stepnumber of the predictor method must be one step more eminent than that of the corrector method.
Step 4: Posit the chief local truncation errors of both predictor-corrector methods.

Step 5: Set the convergence criteria (tolerance level).

Step 6: Input the predictor-corrector method in any mathematical language.

Step 7: Use any one step method to bring forth starting economic values if needed, if not disregard step 7 and

advance to step 8 .

Step 8: Implement the $P(E C)_{m}$ or $P(E C)_{m} E$ mode at $m$ step.

Step 9: If step 8 fails to converge, reiterate the procedure once more and divide the step size (h) by 2 from step 0 or if not, continue to step 10.

Step 10: Valuate the maximum errors after convergency is attained.

Step 11: Print maximum errors.

Step 12: Utilize this formula expressed below to formulate a new step size since converge is reached.

$$
w h=\left|\frac{\varepsilon}{2\left(C_{p+1}^{*}-C_{p+1}\right)}\right|^{\frac{1}{4}}
$$

\section{CONCLUSION}

Mathematical final results have certified the MI is achieved with the assistance of the convergence criteria. This convergence criteria (tolerance level) adjudicates whether the result is admitted or reiterated. The final results establish that MI's performance is observed to yield a better maximum errors than the AS-SCMM, AOSZ-SM, AAFO-SPIBM, FSBM, AEZSNM and DSOIVP as sited in [1, 8-10, 12-14]. Therefore, it can be resolved that the devised method is suitable for solving non-stiff and stiff ODEs.

\section{ACKNOWLEDGMENT}

Authors would like to express their gratitude to Covenant University for their financial backing through grants during the study period.

\section{REFERENCES}

[1] J. R. Dormand, Numerical Methods for Differential Equations, Approach. CRC Press, 1996

[2] J. G. Oghonyon, S. A. Okunuga, S. A. Iyase, "Milne's implementation on block predictor-corrector methods", Journal of Applied Sciences, Vol. 16, No. 5, pp. 236-241, 2016

[3] J. G. Oghonyon, S. A. Okunuga, N. A. Omoregbe, O. O. Agboola, "Adopting a variable step size approach in implementing implicit block multistep method for non-stiff ODEs", Journal of Engineering and Applied Sciences, Vol. 10, No. 2, 174-180, 2015

[4] O. Akinfenwa, S. N. Jator, N. M. Yao, "Continuous block backward differentiation formula for solving stiff ordinary differential equations", Computers and Mathematics with Applications, Vol. 65, No. 7, pp. 9961005,2013

[5] M. K. Jain, S. R. K. Iyengar, R. K. Jain, Numerical Methods for Scientific and Engineering Computation, New Age International (P) Ltd., 2007

[6] J. D. Lambert, Computational Methods inOrdinary Differential Equations, John Wiley and Sons, 1973

[7] S. Mehrkanoon, Z. A. Majid, M. Suleiman, "A variable step implicit block multistep method for solving first-order ODEs", Journal of 
Computational and Applied Mathematics, Vol. 233, No. 9, pp. 23872394, 2010

[8] O. Adesanya, A. A. Momoh, A. M. Alkali, A. Tahir, "Five steps block method for the solution of fourth order ordinary differential equations", International Journal of Engineering Research and Applications, Vol. 2, No. 5, pp. 991-998, 2012

[9] D. O. Awoyemi, S. J. Kayode, L.O . Adoghe, “A five-step p-stable method for the numerical integration of third order ODEs", American Journal of Computational Mathematics, Vol. 4, No. 3, pp. 119-126, 2014

[10] D. O. Awoyemi, S. J. Kayode, L. O. Adoghe, "A six-step continuous multistep method for the solution of general fourth order IVP of ODEs", Journal of Natural Sciences Research, Vol. 5, No. 5, pp. 131-138, 2015

[11] M. K. Duromola, "An accurate five off-step points implicit block method for direct solution of fourth order differential equations", Open Access Library Journal, Vol. 3, No. 6, pp. 1-14, 2016

[12] S. J. Kayode, "An Efficient zero-stable numerical method for fourthorder differential equations", International Journal of Mathematics and Mathematical Sciences, Vol. 2008, Article ID 364021, 2008

[13] S. J. Kayode, "An order six zero-stable method for direct solution of fourth order ODEs", American Journal of Applied Sciences, Vol. 5, No. 11, pp. 1461-1466, 2008

[14] S. J. Kayode, M. K. Duromola, B. Bolaji, "Direct Solution of initial value problems of fourth order ODEs using modified implicit hybrid block method", Journal of Scientific Research and Reports, Vol. 3, No. 21, pp. 2792-2800, 2014

[15] T. Olabode, T. J. Alabi, "Direct block predictor-corrector method for the solution of general fourth order ODEs", Journal of Mathematics Research, Vol. 5, No. 1, pp. 26-33, 2013

[16] Z. B. Ibrahim, K .I. Othman, M. Suleiman, "Implicit r-point block backward differentiation formula for solving first-order stiff ODEs", Applied Mathematics and Computation, Vol. 186, No. 1, pp. 558-565, 2007

[17] I Zarina, I. O. Khairil, M. Suleimann, "Variable step block backward differentiation formula for solving first-order stiff ODEs", World Congress on Engineering, London, UK, Vol. 2, pp. 2-6, July 2-4, 2007

[18] Z. A. Majid, M. B. Suleiman, "Implementation of Four-Point Fully Implicit Block Method for Solving Ordinary Differential Equations", Applied Mathematics and Computation, Vol. 184, No. 2, pp. 514-522, 2007

[19] Z. A. Majid, M. Suleiman, "Parallel direct integration variable step block method for solving large system of higher Order Ordinary Differential Equations", World Academy of Science, Engineering and Technology, Vol. 2, No. 4, pp. 269-273, 2008

[20] J. D. Lambert, Numerical Methods for Ordinary Differential Systems: The Initial Value Problem, John Wiley and Sons, 1991

[21] J. D. Faires, R. L. Burden, Initial-value problems for ODEs, Dublin City University, 2012

[22] C. W. Gear, Numerical Initial Value Problems in ODEs (Automatic Computation), Prentice-Hall, Inc., 1971

[23] L. I. Ken, I. F. Ismail, M. Suleiman, Block Methods for Special Second Order Ordinary Differential Equations, Academic Publishing, 2011

[24] U. M. Ascher, L. R. Petzold, Computer methods for ordinary differential equations and differential algebraic equations. SIAM, Philadelphia, 1998 